\title{
Pros and Cons of Integrating Versus Counting with a Direct Electron Detecting Camera
}

\author{
Joshua Mendez ${ }^{1}$, and Scott Stagg 2,3 \\ 1. Department of Physics, Florida State University, Tallahassee, FL, USA \\ 2. Department of Chemistry and Biochemistry, Florida State University, Tallahassee, FL, USA \\ 3. Institute of Molecular Biophysics, Florida State University, Tallahassee, FL, USA
}

Direct electron detectors (DEDs) were one of the key factors that helped facilitate the transformation of cryogenic electron microscopy (cryo-EM) from "blobology" to being able to model the atomic structures of biological molecules ab initio directly from cryo-EM reconstructions. Broadly speaking, most current DEDs work using complementary metal oxide semiconductor (CMOS) technology where electrons passing through the detector's sensing layer deposit some amount of energy while passing through the chip (1). That energy is converted to a signal, and the signal from an array of pixels combine to produce an image. There are essentially two different methods for producing an image from the CMOS signal: integrating mode where the signal (interpreted as an intensity value) is summed up as multiple electrons pass through a given pixel, or counting mode where individual electron hits are "counted", and the counts are summed up over multiple electron hits. Typically, integrating mode images can be collected much faster, because counting mode requires relatively low electron flux in order to detect individual electron hits. On the other hand, counting mode eliminates the so-called Landau noise resulting from different electrons depositing different amounts of energy, thus, counting mode results in much higher image quality as measured by detective quantum efficiency (DQE) measurements (2). Integrating mode allows for higher-throughput while counting mode allows for better images particularly in the low-resolution portion of a DQE curve. We will report on the pros and cons of counting versus integrating for cryo-EM on a Direct Electron DE64 direct detector using different biological samples. We have implemented both counting and integrating modes of image collection into the Leginon/Appion data collection and processing packages $(3,4)$, and we will discuss differences in throughput and resolution for counting and integrating imaging with different samples.

In integrating mode, the DE64 produces an $8192 \times 8192$ pixel image with a user specified frame rate up to 45 frames per second. With integrating mode, images are typically taken with 1.2 second exposures to generate a $\sim 60 \mathrm{e}^{-} / \AA^{2}$ image. In counting mode, the camera uses hardware binning to increase the frame rate up to 145 frames per second producing 4096 x 4096 pixel images. The frame rate speed-up with hardware binning enables counting over a shorter exposure time, however it is still much slower than integrating; a $56 \mathrm{e}^{-} / \AA^{2}$ image in counting mode requires an exposure of 26 seconds. Despite being slower, the counting images have much improved quality as determined by calculating the modulation transfer function and DQE for the DE64 in both integrating and counting modes (5) (Fig. 1). In future studies, we will characterize the impact the differences in throughput and image quality have on cryo-EM reconstructions of biological samples. 


\section{References:}

McMullan, G. et al, Ultramicroscopy 109 (2009), p. 1144.

McMullan, G. et al, Ultramicroscopy 147 (2014), p. 156.

Suloway, C. et al, Journal of Structural Biology 151 (2005), p. 41.

Lander, G. C. et al, J Struct Biol. 166 (2009), p. 95.

Ruskin, R. S., Yu, Z., and Grigorieff, N. Journal of Structural Biology. 184 (2013), p. 385.

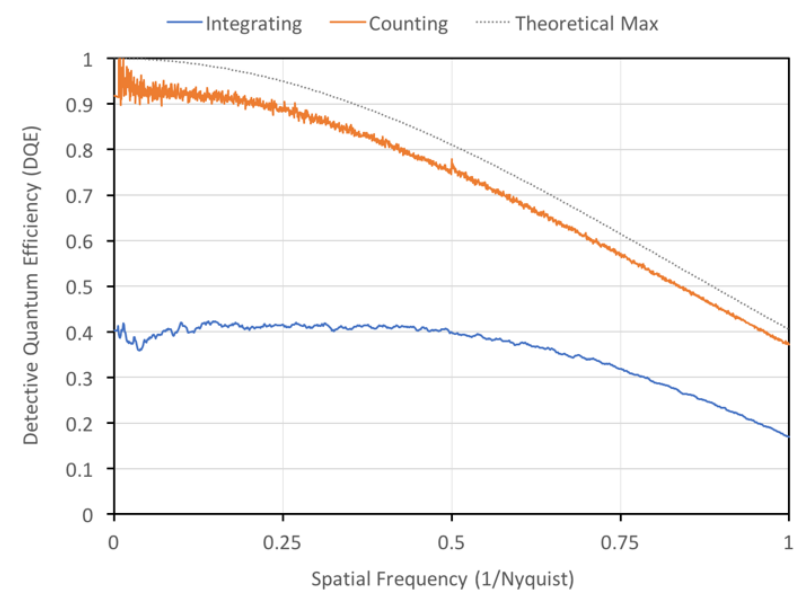

Figure 1. DQE measurements for counting and integrating modes provided by Direct Electron

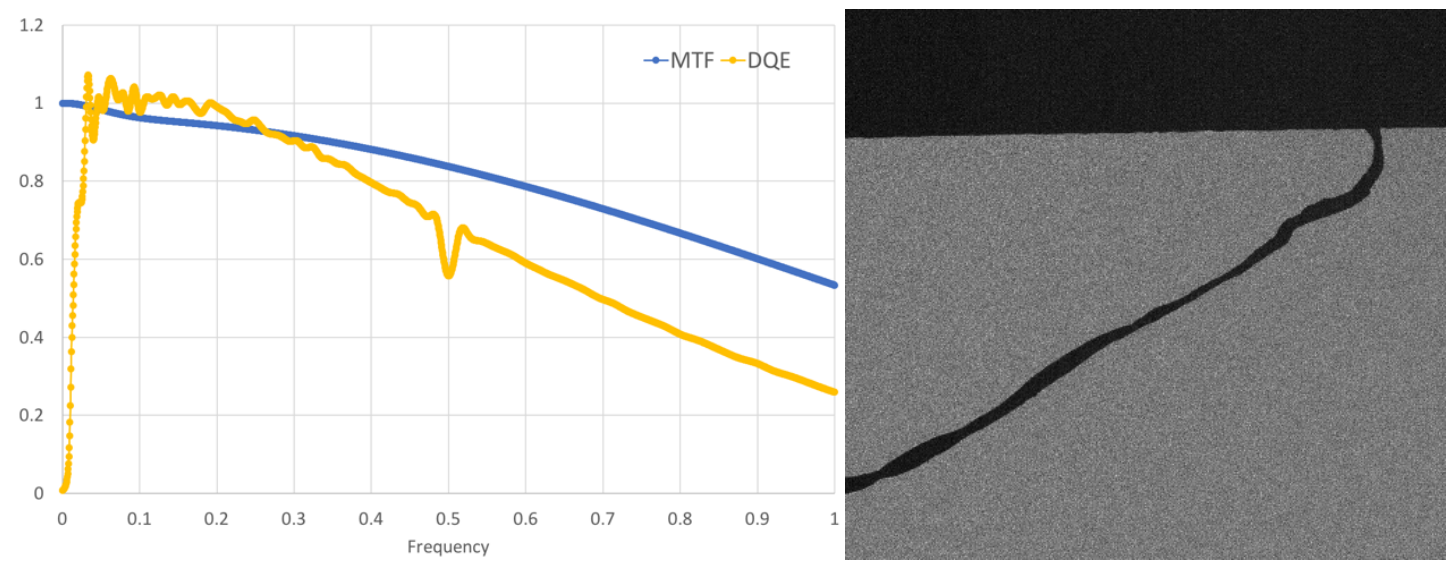

Figure 2. (Left) DQE measurements for counting determined from FindDQE on our instrument. Some artifacts are present, and the noise power spectrum is likely underestimated. (Right) Image of the TEM pointer showing sharp edges indicative of a high quality image. 